\title{
Analysis for Pull-In Voltage of a Multilayered Micro-Bridge Driven by Electrostatic Force
}

\author{
Yu LIU ${ }^{1,2,3}$, Guochao WANG ${ }^{1,2,3}$, Hongyun YANG ${ }^{1,2,3}$ \\ ${ }^{1}$ Automobile College, Chongqing University of Technology, Chongqing, China \\ ${ }^{2}$ Key Laboratory of Automobile Parts \& Test Technique in Chongqing, Chongqing, China \\ ${ }^{3}$ Chongqing Engineering Research Center for Automobile Power System and Control, Chongqing, China \\ Email: liuyu_cq@126.com \\ Received August 8, 2009; revised September 4, 2009; accepted September 12, 2009
}

\begin{abstract}
A trial solution for bending deflection of a multilayered micro-bridge subject to a voltage induced load is presented. The relation between the applied voltage and the displacements of the micro-bridge in the pull-in state is analyzed by energy method. Furthermore, two analytical expressions about normalized displacement and pull-in voltage are carried out. It's proved that the value of normalized displacement is not influenced by residual stress if axial and shear deformation is ignored. Finally, the theoretical results are compared with that of FEM, and they show good agreement.
\end{abstract}

Keywords: MEMS, Electrostatic Actuation, Multilayered Micro-Bridge, Trial Solution, Energy Method, Pull-In Voltage

\section{Introduction}

Moving part is the most frequently used one in MEMS structures. There are various principles that can be used to drive the moving part, including electrostatic, piezoelectric, thermal, magnetism, etc. Compared with the others, the method of electrostatic force drive is more attractive $[1,2]$ because of its larger force caused by micro-effect and non-contact, which is beneficial to high precision. Furthermore, the process of MEMS devices driven by electrostatic force is compatible with IC process.

Micro-bridge structure driven by electrostatic force is familiar in MEMS devices, such as changeable capacitors, RF switches, micro-resonators, pressure sensors, and so on. On one hand, it is a common structure being used to obtain the mechanical parameters of film, just like Young's Modulus, residual stress, yield strength and bending strength [3-5]. But on the other hand, MEMS devices with micro-bridge structure are often with low reliability and worse quality, which is induced by pull-in phenomena. So it's worthy to research the pull-in phenomena of micro-bridge structure subject to a voltage induced load, which is important to MEMS design and optimization. For example, drive voltage must be less than pull-in voltage for most structures driven by electrostatic force, while it's opposite for DMD and selfmeasure unit of micro-accelerometer [6].
Although many people study the pull-in phenomena of the monolayered micro-bridge structure driven by electrostatic force, and get some valuable conclusions, they, however, cannot be applied to the multilayered microbridge structure. So some people [7] devote themselves to research on the pull-in phenomena of the multilayered micro-bridge structure. Whereas the analytical expression is complicated, this depends on the introduction of the assumptions and the form of the trial solution.

This paper presents a trial solution for bending deflection of a multilayered micro-bridge subject to a voltage induced load. The relation between the applied voltage and the displacements of the micro-bridge in the pull-in state is analyzed by energy method. Furthermore, two analytical expressions about normalized displacement and pull-in voltage are carried out. It's proved that the value of normalized displacement is not influenced by residual stress if axial and shear deformation is ignored. Finally, the theoretical results are compared with that of FEM, and they show good agreement.

\section{Model}

The micro-bridge with a rectangular cross-section is made of $\mathrm{n}$ layer materials, illustrated in Figure 1. Here we assume the top of the beam is a conductor, while the others are dielectric. The electrode under the beam is 


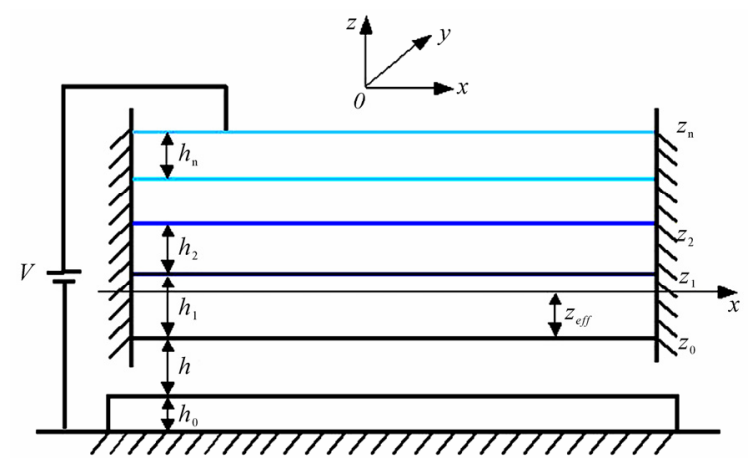

Figure 1. Schematic picture of a multilayered micro-bridge subject to a voltage induced load.

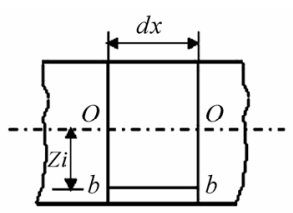

(a)

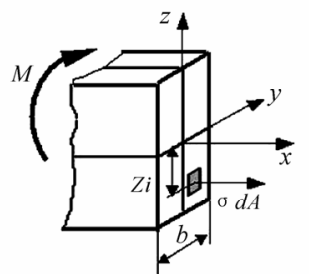

(c)

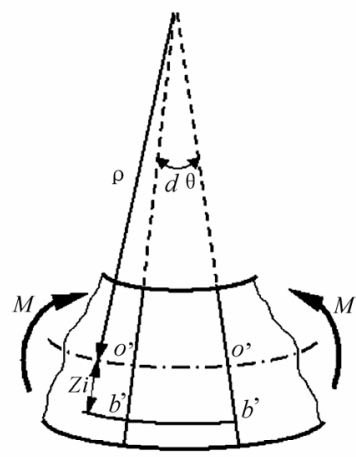

(b)
Figure 2. Deformation of an infinitesimal line element $d x$.

covered with the insulated material, which is fixed on the substrate. It's not difficult to imagine that micro-bridge will be bended when the voltage is added to the beam and the electrode.

Let's explain the meanings of some symbols firstly, where $l$ is the length of the beam. $h_{i}, E_{i}, \varepsilon_{r i}$ are the

thickness, Young's Modulus and relative permittivity of the $i$-layer material respectively. $h_{0}, \varepsilon_{r 0}$ are the thickness and relative permittivity of the insulated layer above the electrode respectively. $V$ is the voltage added to the beam and the electrode. $h$ is the gap distance between the bottom of the beam and the top of the electrode when $V=0$.

Set symmetric axis of beam's cross section as $z$ axis, and positive direction is upwards. Set the equivalent neutral axis as $y$ axis, and its position is needed to be confirmed. Then $x$ axis is the perpendicular axis of $y z$ plane and through the origin. The distance from the equivalent neutral axis to the bottom of the beam is $z_{\text {eff }}$.

It's clear that $z_{\text {eff }} \neq \frac{1}{2} \sum_{i=1}^{n} h_{i}$ due to the different Young's Modulus of n layer materials.
As shown in Figure 2, according to the plane crosssection assumption on pure bending, we calculate the strain $\varepsilon_{i}$ of the fiber bb while $V \neq 0$, which is in the $i$-layer and the primary length as well as the gap distance to neutral surface are $d x, z_{i}$ respectively.

$$
\varepsilon_{i}=-\frac{z_{i}}{\rho}
$$

where $\rho$ is curvature radius of the neutral surface.

Based on the assumption on pure bending, that is, there is no normal stress between the longitudinal fibers; we calculate the stress $\sigma_{i}$ of the fiber bb according to Hooke's law. Of course, the deformation is at the elastic deformation period.

$$
\sigma_{i}=E_{i} \varepsilon_{i}=-E_{i} \frac{z_{i}}{\rho}
$$

Uncountable micro internal force $\sigma_{i} d A$ composes a system of parallel force in space, which can be simplified as three internal force components. What we're concerned about is the component parallel to $x$-axis, namely axial force $N=\int_{A} \sigma_{i} d A$. Based on the equilibrium of static equilibrium $\sum X=N=0$ and the following formula,

$$
\begin{aligned}
N & =\int_{A} \sigma_{i} d A \\
& =\int_{A_{1}} \sigma_{1} d A_{1}+\int_{A_{2}} \sigma_{2} d A_{2}+\cdots+\int_{A_{n}} \sigma_{n} d A_{n} \\
& =-\frac{1}{\rho}\left[\int_{A_{1}} E_{1} z_{1} d A_{1}+\int_{A_{2}} E_{2} z_{2} d A_{2}+\cdots+\int_{A_{n}} E_{n} z_{n} d A_{n}\right] \\
& =-\frac{1}{\rho}\left[\int_{-z_{\text {eff }}}^{h_{1}-z_{\text {eff }}} E_{1} b z_{1} d z_{1}+\int_{h_{1}-z_{\text {eff }}}^{h_{1}+h_{2}-z_{\text {eff }}} E_{2} b z_{2} d z_{2}+\cdots+\int_{h_{1}+h_{2}+\cdots+h_{n-1}-z_{\text {eff }}}^{h_{1}+h_{1}+\cdots+h_{n}-z_{\text {eff }}} E_{n} b z_{n} d z_{n}\right]
\end{aligned}
$$

We get

$$
z_{\text {eff }}=\frac{\sum_{j=1}^{n} E_{j} h_{j}\left(\frac{h_{j}}{2}+\sum_{i=1}^{j-1} h_{i}\right)}{\sum_{i=1}^{n} E_{i} h_{i}}
$$

Therefore,

$$
\begin{gathered}
z_{0}=-z_{\text {eff }} \\
z_{i}=\left(\sum_{j=1}^{i} h_{j}\right)-z_{\text {eff }} \quad i=1,2, \cdots, n
\end{gathered}
$$

where $z_{0}, z_{n}$ are the coordinates of the micro-bridge's top and bottom surface along $z$-axis, while $z_{1}, \cdots, z_{n-1}$ are that of layer-layer interface respectively, as shown in Figure 1.

\section{Solution}

Here we choose a trial solution for bending deflection of 
a multilayered micro-bridge subject to a voltage induced load as following [8]:

$$
w(x)=A\left(1-\cos \frac{2 \pi x}{l}\right)=2 A \sin ^{2} \frac{\pi x}{l}
$$

where $A$ is the undetermined coefficient and $2 A$ is the maximum deflection of the micro-bridge.

It's acceptable to neglect the influence of axial and shear deformation on bending due to the small displacement assumption of the micro-bridge.

\subsection{No Consideration of Residual Stress}

For this situation, the strain $\varepsilon_{i}$ of the $i$-layer material only includes flexural strain $\varepsilon_{b i}$. That is,

$$
\varepsilon_{i}=\varepsilon_{b i}=-z \frac{d^{2} w}{d x^{2}}=z \frac{4 \pi^{2} A}{l^{2}} \cos \frac{2 \pi x}{l}
$$

So the unit volume deformation energy and the deformation energy of the $i$-layer material are described by the following formula:

$$
\begin{gathered}
u_{i}=\frac{1}{2} E_{i} \varepsilon_{i}^{2} \\
U_{s i}=\int_{v_{i}} u_{i} d v_{i}=\frac{E_{i} b}{2} \int_{0}^{l} \int_{z_{i-1}}^{z_{i}} \varepsilon_{i}^{2} d z d x
\end{gathered}
$$

Therefore, the total deformation energy of the micro-bridge $U_{s}$ is equal to the summation of the deformation energy of $\mathrm{n}$ layer materials. That is,

$$
\begin{array}{r}
U_{s}=\sum_{i=1}^{n} U_{s i}=\sum_{i=1}^{n} \frac{E_{i} b}{2} \int_{0}^{l} \int_{z_{i-1}}^{z_{i}} \varepsilon_{i}^{2} d z d x \\
=\frac{b l A^{2}}{12}\left(\frac{2 \pi}{l}\right)^{4} \sum_{i=1}^{n} E_{i}\left(z_{i}^{3}-z_{i-1}^{3}\right)
\end{array}
$$

Considering the influence of the insulated layers on the gap distance, we introduce the equivalent gap distance $\tilde{h}_{\text {[9] }}$

$$
\tilde{h}=h+\frac{h_{0}}{\varepsilon_{r 0}}+\frac{h_{1}}{\varepsilon_{r 1}}+\cdots+\frac{h_{n-1}}{\varepsilon_{r(n-1)}}
$$

where $h_{i} / \varepsilon_{r i}$ is the equivalent air gap distance of the $i$-layer insulated material. Then the model in Figure 1 is simplified as Figure 3, where $\tilde{\delta}$ is the equivalent thickness, which will be discussed in detail later.

So the electrostatic energy can be described as [8]

$$
U_{e}=-\frac{V^{2} b \varepsilon_{0} l}{2 \sqrt{\tilde{h}(\tilde{h}-2 A)}}
$$

where $\varepsilon_{0}$ is the permittivity of vacuum.

Therefore, the energy function is described as [10]

$$
\Pi=U_{s}+U_{e}
$$

Because the value of the electrostatic force is in inverse proportion to the square of distance, and the distribution of electric field is influenced by structural dimension as well as position, so the issue of bending equilibrium stability should be considered. It exists when the electrostatic force is equal to the elastic force. If the voltage $V$ exceeds the critical value, the equilibrium state is destroyed and the micro-bridge will contact with the fixed plane, which is called pull-in phenomena. The minimum critical voltage is called pull-in voltage, marked as $V_{\mathrm{PI}}$. Here,

$$
\frac{\partial \Pi}{\partial A}=0 \quad \text { and } \quad \frac{\partial^{2} \Pi}{\partial A^{2}}=0
$$

Mark the maximum deflection of the micro-bridge under $V_{\mathrm{PI}}$ as $2 A_{c r}$, then

$$
\begin{aligned}
& \frac{A_{c r}}{6}\left(\frac{2 \pi}{l}\right)^{4} \sum_{i=1}^{n} E_{i}\left(z_{i}^{3}-z_{i-1}^{3}\right)=\frac{V^{2} \varepsilon}{2} \tilde{h}\left(\tilde{h}^{2}-2 \tilde{h} A_{c r}\right)^{-\frac{3}{2}} \\
& \frac{1}{6}\left(\frac{2 \pi}{l}\right)^{4} \sum_{i=1}^{n} E_{i}\left(z_{i}^{3}-z_{i-1}^{3}\right)=\frac{3 V^{2} \varepsilon}{2} \tilde{h}^{2}\left(\widetilde{h}^{2}-2 \tilde{h} A_{c r}\right)^{-\frac{5}{2}}
\end{aligned}
$$

So

$$
\frac{A_{c r}}{\tilde{h}}=0.2
$$

And the normalized displacement

$$
\beta=\frac{2 A_{c r}}{\tilde{h}}=0.4
$$

\subsection{Consideration of Residual Stress}

For this situation, the strain $\varepsilon_{i}$ of the $i$-layer material includes not only flexural strain $\varepsilon_{b i}$ but also the strain

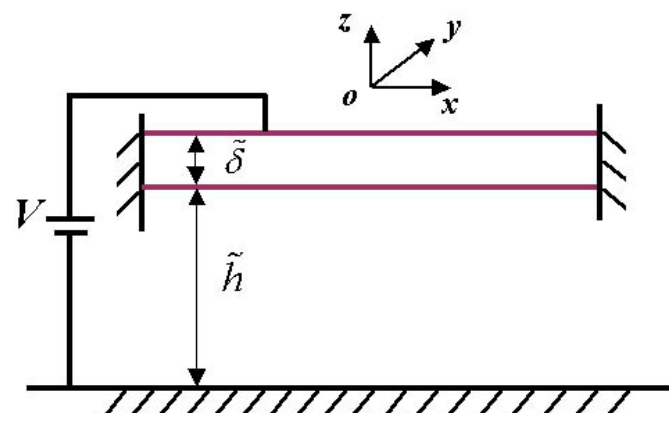

Figure 3. The equivalent model of a multilayered microbridge. 
induced by the residual stress $\sigma_{i}$ of the $i$-layer material. That is,

$$
\varepsilon_{i}=\varepsilon_{b i}+\frac{\sigma_{i}}{E_{i}}=-z \frac{d^{2} w}{d x^{2}}+\frac{\sigma_{i}}{E_{i}}=-z \frac{4 \pi^{2} A}{l^{2}} \cos \frac{2 \pi x}{l}+\frac{\sigma_{i}}{E_{i}}
$$

Similarly, the deformation energy of the micro-bridge

$$
\begin{aligned}
U_{s} & =\sum_{i=1}^{n} \frac{E_{i} b}{2} \int_{0}^{l} \int_{z_{i-1}}^{z_{i}} \varepsilon_{i}^{2} d z d x \\
& =\frac{b l A^{2}}{12}\left(\frac{2 \pi}{l}\right)^{4} \sum_{i=1}^{n} E_{i}\left(z_{i}^{3}-z_{i-1}^{3}\right)+\sum_{i=1}^{n} \frac{b l h_{i} \sigma_{i}^{2}}{2 E_{i}}
\end{aligned}
$$

Because the second part of Formula (5) is not the function of the undetermined coefficient $A$, so the results from Formula (1) are same to that of Subsection 3.1.

It's clear that the value of normalized displacement is not influenced by residual stress if axial and shear deformation is ignored.

Based on Equations (2) and (4), we get

$$
V_{P I}=\sqrt{48.290 \frac{\tilde{h}^{3} \sum_{i=1}^{n} E_{i}\left(z_{i}^{3}-z_{i-1}^{3}\right)}{\varepsilon_{0} l^{4}}}
$$

For the multilayered micro-bridge with a rectangular cross-section, the area moment of inertia

$$
\widetilde{I}=\int_{z_{0}}^{z_{n}} b z^{2} d z=\frac{b}{3}\left(z_{n}^{3}-z_{0}^{3}\right)
$$

and the flexural stiffness

$$
\widetilde{E I}=\sum_{i=1}^{n} \int_{z_{i-1}}^{z_{i}} E_{i} b z^{2} d z=\frac{b}{3} \sum_{i=1}^{n} E_{i}\left(z_{i}^{3}-z_{i-1}^{3}\right)
$$

then the equivalent Young's Modulus

$$
\widetilde{E}=\frac{\widetilde{E I}}{\widetilde{I}}=\frac{\sum_{i=1}^{n} E_{i}\left(z_{i}^{3}-z_{i-1}^{3}\right)}{z_{n}^{3}-z_{0}^{3}}
$$

and the equivalent thickness

$$
\tilde{\delta}=\left(\frac{12 \tilde{I}}{b}\right)^{\frac{1}{3}}=\left[4\left(z_{n}^{3}-z_{0}^{3}\right)\right]^{\frac{1}{3}}
$$

Therefore, the formula (6) is simplified as

$$
V_{P I}=\sqrt{12.0725 \frac{\tilde{E} \tilde{\delta}^{3} \tilde{h}^{3}}{\varepsilon_{0} l^{4}}}
$$

It's clear that the multilayered micro-bridge can be considered as a monolayered micro-bridge if only the equivalent Young's Modulus $\tilde{E}$ and the equivalent thickness $\tilde{\delta}$ are carried out.

In the above discussion, we assume the top of the beam is a conductor while the others are dielectric. If the conductor is not on the top of the micro-bridge, or more than one layer is conductor, the equivalent gap distance $\tilde{h}$ will differ.

\section{Validation}

All the above deduction is based on a given trial solution for bending deflection. Are they correct? How about the accuracy? Now we try to prove it by simulation.

Firstly, some examples relating to the monolayered micro-bridges are considered. For the monolayered micro-bridge given in paper [11], the value of $V_{\mathrm{PI}}$ is $32.5 \mathrm{~V}$, and the simulated values by ANSYS as well as Intellisuite are $37.6 \mathrm{~V}, 39.5 \mathrm{~V}$ respectively, which are presented in paper [11]. Whereas the analytical value based on the Formula (7) is $34.2 \mathrm{~V}$, which is more close to the simulated values. And for that given in paper [12], the simulated value of $V_{\mathrm{PI}}$ is $40 \mathrm{~V}$, while the analytical value based on the Formula (7) is $40.99 \mathrm{~V}$. The error is only $2.48 \%$.

Secondly, a multilayered micro-bridge with a rectangular cross-section is considered. It's made of two layer materials. And the electrode under it is covered with $0.5 \mu \mathrm{m} \quad \mathrm{Si}_{3} \mathrm{~N}_{4}$.

Model 1: the top material is gold. And the other is $\mathrm{Si}_{3} \mathrm{~N}_{4}$.

Model 2: the top material is $\mathrm{Si}_{3} \mathrm{~N}_{4}$. And the other is gold.

Table 1. Geometry and material parameters of the bi-layered micro-bridge.

\begin{tabular}{lcccc}
\hline \multicolumn{1}{c}{ Item } & Symbol (unit) & $\begin{array}{c}\text { Model } \\
1\end{array}$ & Model 2 \\
\hline length & $l(\mu \mathrm{m})$ & \multicolumn{2}{c}{400} \\
width & $b(\mu \mathrm{m})$ & \multicolumn{2}{c}{50} \\
gap distance $(V=0)$ & $h(\mu \mathrm{m})$ & \multicolumn{2}{c}{2.0} & \multicolumn{2}{c}{0.5} \\
thickness of the & $h_{0}(\mu \mathrm{m})$ & \multicolumn{2}{c}{0.5} \\
insulated layer & $h_{1}(\mu \mathrm{m})$ & 2.0 & & 2.0 \\
thickness & $h_{2}(\mu \mathrm{m})$ & 0.5 & & \\
relative permittiv- & $\varepsilon_{r 0}=\varepsilon_{r 1}$ & & 8.0 & \\
ity & $E_{1}(\mathrm{GPa})$ & 210 & & 57 \\
Young's Modulus & $E_{2}(\mathrm{GPa})$ & 57 & 210 \\
\hline
\end{tabular}

Table 2. $V_{P I}, \beta$ of the bi-layered micro-bridge.

\begin{tabular}{ccccc}
\hline Item & Model & Analytical values & Simulated values & Error \\
\hline \multirow{2}{*}{$V_{\mathrm{PI}}$} & model 1 & $37.9671 \mathrm{~V}$ & $40.2187 \mathrm{~V}$ & $-5.60 \%$ \\
& model 2 & $31.9798 \mathrm{~V}$ & $33.3438 \mathrm{~V}$ & $-4.09 \%$ \\
& model 1 & & 0.4069 & $-1.70 \%$ \\
$\beta$ & model 1 & \multirow{2}{*}{0.4} & 0.3933 & $1.70 \%$ \\
& model 2 & & 0.3972 & $0.70 \%$ \\
& model 2 & 0.4132 & $-3.19 \%$ \\
\hline Note:
\end{tabular}

Note: \# Residual stress of the gold layer is $100 \mathrm{MPa}$. 
The geometry and material parameters of the bi-layered micro-bridge are listed in Table 1. The micro-bridge will be bended when subject to a voltage induced load.

For Models 1 and 2, we get the simulated values of the pull-in voltage $V_{\mathrm{PI}}$ and the normalized displacement $\beta$ by FEM. Table 2 lists the simulated performances and the analytical values of $V_{\mathrm{PI}}$ and $\beta$, as well as the error of them. It shows good correlation. So the trial solution of deflection is acceptable.

\section{Conclusions}

This paper presents a trial solution for bending deflection of a multilayered micro-bridge subject to a voltage induced load. The relation between the applied voltage and the displacements of the micro-bridge in the pull-in state is analyzed by energy method. Furthermore, two analytical expressions about normalized displacement and pull-in voltage are carried out. The theoretical results are compared with that of FEM, and they show good agreement.

All results in this paper are based on the form of the selected trial solution. The better the selected trial solution is, the higher the accuracy of solution is. Moreover, the axial and shear deformation is ignored, which maybe has influence on the stress and strain. Besides, in order to get an analytical expression of $V_{\mathrm{PI}}$, it's difficult to consider the influence of some secondary effects, such as the marginal effect of electric field. However, this paper provides an analytical model with high accuracy for a multilayered micro-bridge driven by electrostatic force, which is beneficial to design, optimization and application of MEMS devices with the micro-bridge structure.

\section{References}

[1] H. Y. Ma, Y. H. Wang, M. L Wang, et al. "The sequential coupling analysis of a cantilever driving by electrostatic force in an optical switch," Optical Instruments, Vol. 25, No. 3, pp. 17, 2003.
[2] G. Li and N. R. Aluru, "Efficient mixed-domain analysis of electrostatic MEMS," ICCAD, pp. 474, 2002.

[3] T. Y. Zhang, Y. J. Su, C. F. Qian, et al. "Micro-bridge testing of silicon nitride thin films deposited on silicon wafers," Acta Mater, Vol. 48, pp. 2843, 2000.

[4] Y. Zhou, C. S. Yang, J. A. Chen, et al. "Investigation of Young's modulus and residual stress of copper film microbridges by MEMS technology," Modern Scientific Instruments, Vol. 4, pp. 45, 2003.

[5] M. J. Wang, Y. Zhou, J. A. Chen, et al. "Measurements of elastic modulus and residual stress of nickel film by microbridge testing methods," Electronic Components \& Materials, Vol. 23, No. 12, pp. 13, 2004.

[6] H. Yang, M. H. Bao, S. Q. Shen, et al. "The displacement characteristics of the micromechanical structures driven by electrostatic force," Journal of Fudan University (Natural Science), Vol. 38, No. 3, pp. 282, 1999.

[7] H. Rong, Q. A. Huang, M. Nie, et al. "An analytical model for pull-in voltage of doubly-clamped multi-layer beams," Chinese Journal of Semiconductors, Vol. 24, No. 11, pp. 1185, 2003.

[8] D. H. Sun, Y. Q. Huang, W. Zheng, et al. "On the modeling methodology of MEMS system-level simulation," Journal of Xiamen University (Natural Science), Vol. 40, No. 2, pp. 297, 2001.

[9] M. Nie, Q. A. Huang, J. H. Wang, et al. "Analysis of deflection and pull-in voltage of a multi-layer cantilever under an electrostatic load," Chinese Journal of mechanical engineering, Vol. 40, No. 8, pp. 72, 2004.

[10] Y. Y. Wang, T. I. Kamins, B. Y. Zhao, et al. Polysilicon Film and its Application in IC (2nd Version), Science Press, Beijing, 2001.

[11] C. S. Wang, W. B. Zhang, J. Fang, et al. "Research on coupled electro-mechanical analysis and application for typical components in MEMS," Journal of Mechanical Strength, Vol. 23, No. 4, pp. 503, 2001.

[12] P. M. Osterberg, S. D. Senturia, "M-test: A test chip for MEMS material property measurement using electrostatically actuated test structures," Journal of Microelectromechanical Systems, Vol. 6, No. 2, pp. 107, 1997. 